\title{
Reporte del hallazgo de restos de Hilarcotherium sp. (Mammalia, Astrapotheria) y de material asociado en una nueva localidad fosilífera del valle inferior del Magdalena, ciénaga de Zapatosa, Cesar, Colombia
}

\author{
(1) Mauricio Pardo Jaramillo \\ Dirección Técnica de Geociencias Básicas, Museo Geológico e Investigaciones Asociadas, \\ Museo Geológico “José Royo y Gómez”, Servicio Geológico Colombiano, Bogotá, D.C., Colombia
}

\begin{abstract}
Resumen
En abril de 2018 el Museo Geológico “José Royo y Gómez” recibió el reporte del hallazgo de material paleontológico en cercanías del corregimiento de La Mata, en el municipio de Chimichagua (Cesar), a orillas de la ciénaga de Zapatosa. El material recolectado por pobladores del lugar incluía un gran diente de caimán (Purussaurus), restos de tortugas (Podocnemididae?) y matamata (Chelus), y un fragmento de maxilar de Hilarcotherium sp. (Mammalia, Astrapotheria), conocido previamente por los hallazgos de la especie tipo Hilarcotherium castanedaii (VallejoPareja, et al., 2015) en los estratos del Grupo Honda (Formación La Victoria) en el municipio de Purificación, Tolima, y de Hilarcotherium miyou (Carrillo, et al., 2018) de la Formación Castilletes, al sureste de la península de La Guajira. Además, este informe constituye el primer registro de rocas continentales del Mioceno en esta región del país. (C) 2018. Acad. Colomb. Cienc. Ex. Fis. Nat.
\end{abstract}

Palabras clave: Astrapotheria; Hilarcotherium castanedaii; Hilarcotherium miyou; Mioceno.

Report on the finding of remains of Hilarcotherium sp. (Mammalia, Astrapotheria) and associated material in a new fossil locality in the Lower Valley of Magdalena, Zapatosa wetlands, Cesar, Colombia

\begin{abstract}
In April 2018, the "José Royo y Gómez" Geological Museum received a report on the discovery of paleontological material in the vicinity of La Mata village, in the municipality of Chimichagua (Cesar), on the banks of the Zapatosa wetlands. The material collected by local residents included a large alligator tooth (Purussaurus), remains of (Podocnemididae?) and matamata turtles (Chelus), and a maxillary fragment of Hilarcotherium sp. (Mammalia, Astrapotheria) known so far for the findings of the type species Hilarcotherium castanedaii (Vallejo-Pareja, et al., 2015) in the strata of the Honda Group (La Victoria Formation) in the town of Purificación, Tolima, and of Hilarcotherium miyou (Carrillo, et al., 2018) from the Castilletes Formation in the southeast area of La Guajira peninsula. Additionally, this report is the first record of Miocene continental rocks in this region of the country. (C) 2018. Acad. Colomb. Cienc. Ex. Fis. Nat.
\end{abstract}

Key words: Astrapotheria; Hilarcotherium castanedaii; Hilarcotherium miyou; Miocene.

\section{Introducción}

Los estudios geológicos y paleontológicos del norte de Colombia han ganado relevancia en los últimos años. Mediante los trabajos de cartografía geológica en esta región, desarrollados principalmente por el Servicio Geológico Colombiano en asociación con diversas entidades, se ha logrado una cobertura del $100 \%$ de la cartografía en escala 1:100.000 de la costa Atlántica, de la península de La Guajira y los límites nororientales con Venezuela (Planchas 1 a 57). Asimismo, investigadores de la Universidad Nacional han adelantado trabajos en paleontología, entre los cuales es de gran relevancia el de Villarroel \& Clavijo (2005), por su clasificación taxonómica de cinco ejemplares fósiles (Mammalia) y la revisión general de la estratigrafía; en él los autores proponen un modelo más sencillo que agrupa las unidades litoestratigráficas del Neógeno en la costa Caribe. Otros estudios de gran relevancia internacional han sido liderados por el Smithsonian Tropical Research Institute STRI, entre los cuales cabe destacar los estudios del Cerrejón, con descubrimientos tan famosos como el de la Titanoboa

Correspondencia:

Mauricio Pardo Jaramillo; mpardo@sgc.gov.co

Recibido: 26 de junio de 2018

Aceptado: 3 de agosto de 2018

Editor: Carlos Jaramillo Muñoz 
cerrejonensis (Head, et al., 2009), la serpiente más grande de la que se tenga registro a nivel mundial, así como el trabajo multidisciplinario de Moreno, et al. (2015), con prefacio de Jaramillo, et al. (2015), en el cual se revisan y estudian los depósitos del Neógeno en la cuenca de Cocinetas, así como otros estudios paleontológicos de la misma cuenca, como el de Carrillo, et al. (2018), en el que se describe el nuevo astrapoterio Hilarcotherium miyou, entre otros. Se destacan también estudios como el de Moreno-Bernal, et al. (2016) sobre Crocodylia y otros en este mismo contexto, como el de Suárez, et al. (2016), quienes describen la nueva especie de marsupial carnívoro Lycopsis padillai (Mammalia, Metatheria).

Esta nota constituye el primer reporte del hallazgo de material del género Hilarcotherium además del encontrado previamente de su especie tipo Hilarcotherium castanedaii (Vallejo-Pareja, et al., 2015), y de Hilarcotherium miyou (Carrillo, et al., 2018). El material del astrapoterio consta de un fragmento del maxilar y se le asignó el número de catálogo SGC-MGJRG.2018.V.2. Este material, el de un diente de caimán (Purussaurus) y los restos de tortugas (Podocnemididae?) y matamatas (Chelus), constituyen el primer reporte paleontológico en la zona, así como de la identificación de sedimentitas continentales del Mioceno en la región, con edades y facies no reportadas en anteriores trabajos de cartografía de esta zona.

Estos hallazgos hacen parte de los descubrimientos de nuevos especímenes paleontológicos y biológicos que se han hecho recientemente en el país. Son restos fósiles que se encuentran en zonas que no era posible visitar antes del fin del conflicto armado debido a su complicada situación de orden público y su difícil acceso.

\section{Procedencia geográfica y litoestratigráfica de la pieza}

El ejemplar reportado se encontró en una playa a orillas de la ciénaga de Zapatosa en la península (isla en tiempo de invierno) conocida como Llevanzuelo o Isla Barrancones según la plancha geológica $55 \mathrm{El}$ Banco, en escala de 1:100.000 (Ingeominas-UIS, 2006), y situada a $1,9 \mathrm{~km}$ al suroeste del corregimiento de La Mata, del municipio de Chimichagua, departamento del Cesar. Las siguientes son las coordenadas geográficas del punto: $9^{\circ} 07^{\prime} 58,1^{\prime \prime} \mathrm{N}$ y $73^{\circ}$ $45^{\prime} 49,5^{\prime \prime} \mathrm{O}$, a 25 metros sobre el nivel del mar.

El espécimen fue recolectado en febrero del año 2018 por los señores Hasmet Florián y José Martínez, habitantes del corregimiento de La Mata, quienes reportaron el hallazgo y gentilmente donaron las piezas al Museo Geológico "José Royo y Gómez" del Servicio Geológico Colombiano. Los restos fósiles fueron encontrados en tiempo seco en forma de material suelto en las playas originadas por la erosión de los pequeños barrancos ubicados en cercanías de las orillas de la ciénaga.

Las sedimentitas de donde se desprendieron los fósiles están compuestas por areniscas cuarzosas de grano fino y medio, de color rojizo, con algunos clastos de origen metamórfico (cuarcitas) y, en menor proporción, limolitas y arcillolitas rojizas y guijos de origen volcánico, características que sugieren que los estratos podrían atribuirse al Grupo Real, unidad miocénica no reconocida hasta el momento en la plancha 55 El Plato, sin embargo, en dicha plancha se describe el Grupo Real como conformado por areniscas cuarzosas, suelos y sedimentos rojos y niveles volcánicos piroclásicos, en tanto que en la parte superior aparecen capas de conglomerados intercaladas con estratos arcillosos rojos. Así pues, el hallazgo de sedimentitas continentales en varias de las islas de la ciénaga de Zapatosa constituye un aporte que permitirá refinar la cartografía de la región.

\section{Paleontología sistemática}

Clase: Mammalia

Orden: Astrapotheria Lydekker, 1894

Familia: Astrapotheriidae Ameghino, 1887

Subfamilia: Uruguaytheriinae Kraglievich, 1928

Género: Hilarcotherium Vallejo-Pareja, et al., 2015

Especie: Hilarcotherium sp.

\section{Material de referencia y métodos}

Astrapotherium? ruderarium (FMNH 13426); Astrapotherium magnum (FMNH P14251, P13173); Astrapothericulus iheringi (MACN A 52-419, MACN A 52-417), Albertogaudrya (CNS-V-10.000(1-2); Granastrapotherium snorki (UCMP 40017, 40187, 40188, 40358, 40408 IGMp-881230); Parastrapotherium holmbergi (FMNH 13329); Parastrapotherium sp. (FMNH 13569); Xenastrapotherium kraglievichi (MLP 12-96, UCMP 38115); Xenastrapotherium christi (NMB Aa 21).Hilarcotherium castanedaii (IGM p881231), Maddenia (MPEF PV 7735), Astraponotus (MPEF PV 1296 A), Hilarcotherium miyou (IGM p881327, MUN-STRI 34216).

El material de astrapoterio descrito proviene de la ciénaga de Zapatosa, en el departamento del Cesar, nororiente de Colombia (Figura 1 y 2); la terminología de la morfología dental es la usada por Jhonson (1984) y las medidas dentales se hicieron siguiendo la metodología de Jhonson \& Madden (1997), Vallejo-Pareja, et al. (2015) y Carrillo, et al. (2018).

\section{Descripción de la pieza}

Se trata de un fragmento del maxilar izquierdo con un premolar (P4) y un molar (M1). Los dientes superiores en su lado labial presentan hipsodoncia unilateral, lo que significa que exhiben coronas más altas del lado labial que del lado lingual. Se observa la presencia de un bolsillo anterolingual en M1, el parastilo reducido, y la falta de un cíngulo en el lado labial; el reborde o cíngulo presente en el lado lingual permite asignar los restos encontrados a la subfamilia Uruguaytheriinae (Kraglievich, 1928; Johnson \& Madden, 1997; Kramarz \& Bond, 2009, 2011; VallejoPareja, et al., 2015). Al igual que Granastrapotherium (Johnson \& Madden, 1997) y Comahuetherium (Kramarz 


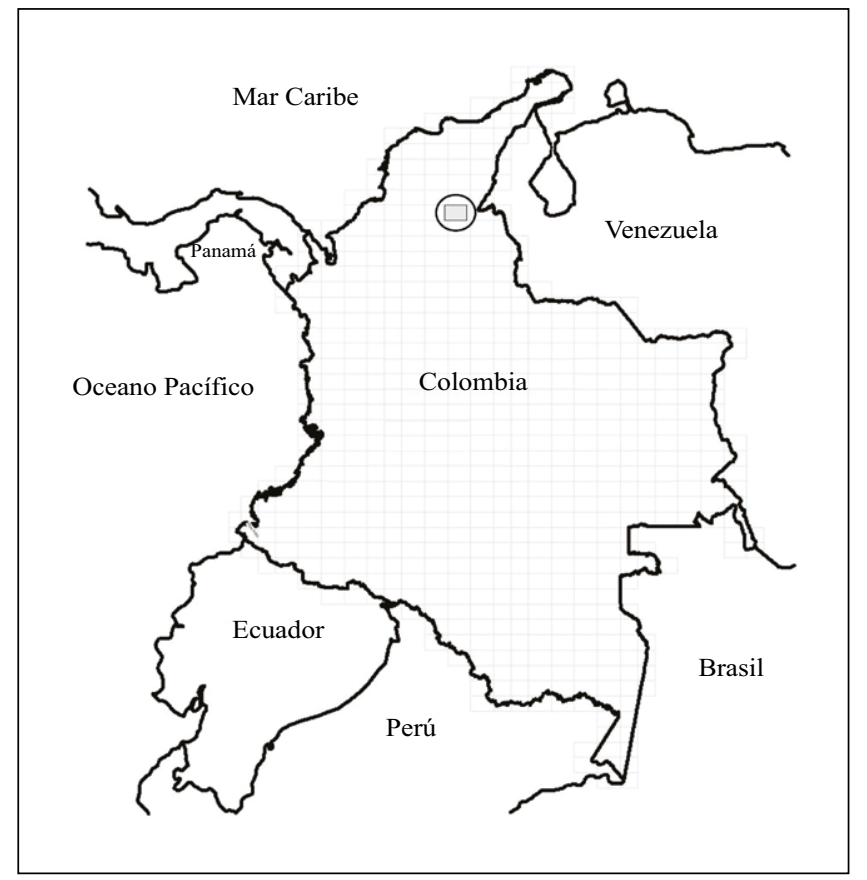

Figura 1. Lugar del hallazgo
\& Bond, 2011), Hilarcotherium (Vallejo-Pareja, et al., 2015; Carrillo, et al., 2018) e Hilarcotherium miyou (Carrillo, et al., 2018), tiene solo un premolar superior (P4), el cual presenta un cíngulo lingual igual al de $H$. castanedaii, aunque también algunas diferencias con el P4 de $H$. castanedaii y de $H$. miyou, entre ellas, la presencia de un bolsillo anterolingual en $H$. castanedaii que en el ejemplar en estudio es apenas incipiente, en tanto que en H. miyou está ausente. El P4 es más pequeño que el M1 (Tabla 1), y representa el $26 \%$ de la longitud total de la serie M1-P4, y tan solo el $15 \%$ del total de la superficie oclusal P4-M1, teniendo en cuenta que falta una parte en el borde bucal. Al igual que en $H$. castanedaii y G. snorki, el P4 entra en oclusión directa con los dientes inferiores, lo cual se evidencia por el desgaste exhibido en su superficie oclusal, la cual está ligeramente por debajo del nivel del M1 al igual que en Granastrapotherium snorki e $H$. miyou. El P4 evidentemente estaba incorporado a la función masticatoria (Figura 3-4).

En el P4 el protocono está bien desarrollado, en tanto que no se conserva el esmalte del lado labial ni la región paraestilar del ectolofo; no hay signos de un pliegue labial como el de Astrapothericulus (Ameghino, 1887,

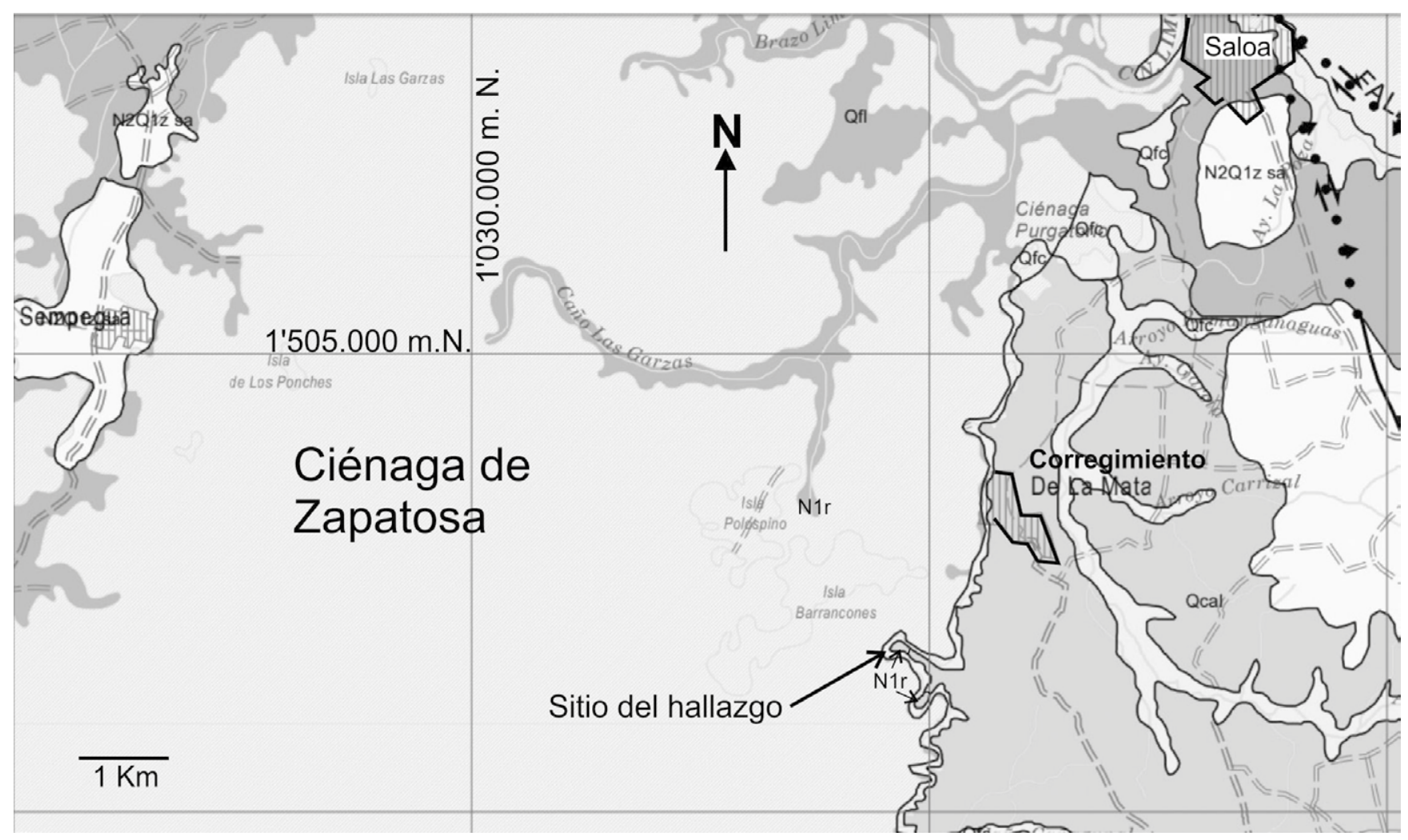

Figura 2. Mapa geológico con el sitio del hallazgo. Detalle; modificado de la plancha geológica 55, escala 1:100.000, El Banco, 2006, Origen Bogotá. Qfc: Depósitos aluviales de canal. Bloques, gravas, arenas y lodos; Qcal: Depósitos de abanicos y terrazas. Bloques, cantos, gravas, arenas y lodos volcano-sedimentarios, ígneas, metamórficas y sedimentarias; N2Q1z sa: Formación ZaAmbrano-Sedimentitas de Arjona. Conjuntos arcillosos de color marrón a violeta, con capas delgadas de areniscas calcáreas fosilíferas; N1r: Grupo Real. Arenas cuarzosas, suelos y sedimentos rojos y niveles volcánicos piroclásticos; en la parte superior, capas de conglomerados intercaladas con estratos arcillosos rojos 
Tabla 1. Medidas dentales: * corona del diente incompleta; ${ }^{* *}$ medida tomada en el alveolo

\begin{tabular}{lccccc}
\hline & $\begin{array}{c}\text { Medidas en } \\
\text { milímetros }\end{array}$ & Lado & $\begin{array}{c}\text { Largo antero- } \\
\text { posterior }\end{array}$ & $\begin{array}{c}\text { Ancho máximo } \\
\text { (bucal-lingual) }\end{array}$ & $\begin{array}{c}\text { Altura máxima de la } \\
\text { corona en el lado bucal }\end{array}$ \\
\hline SGC-MGJRG.2018.V.2 & P4 & Izquierdo & 15,66 & 20,4 & 20,68 \\
\hline IGM p881231 & M1 & Izquierdo & 41,04 & 38,7 & 24,84 \\
& P4 & Izquierdo & 18,95 & 21,23 & 21,15 \\
\hline MUN-STRI 34216 & M1 & & 42,47 & 51,3 & 17,75 \\
& P4 & Izquierdo & $31,8^{* *}$ & $40,7 * *$ & $65,4^{*}$ \\
\hline
\end{tabular}

1904), el de Parastrapotherium (Ameghino, 1887), el de Comahuetherium o el de Maddenia (Kramarz \& Bond, 2009). Al igual que en H. castanedaii, el $\mathrm{P} 4$ no tiene hipocono y posee un pequeño metalofo que se extiende lingualmente desde el protocono a diferencia de Astraponotus

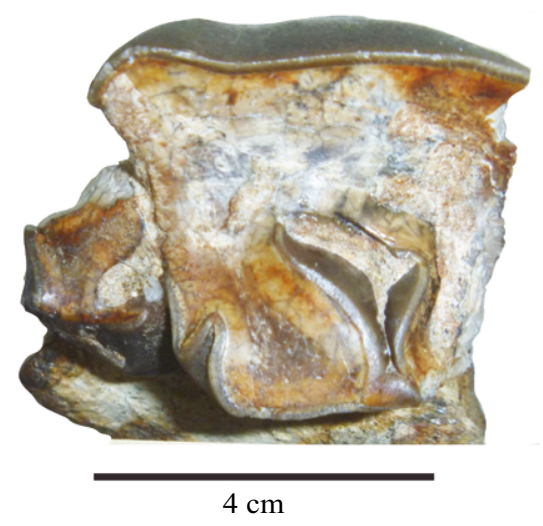

Figura 3. Nomenclatura dental: vista oclusal del premolar (P1) y el molar (M1) superiores izquierdos

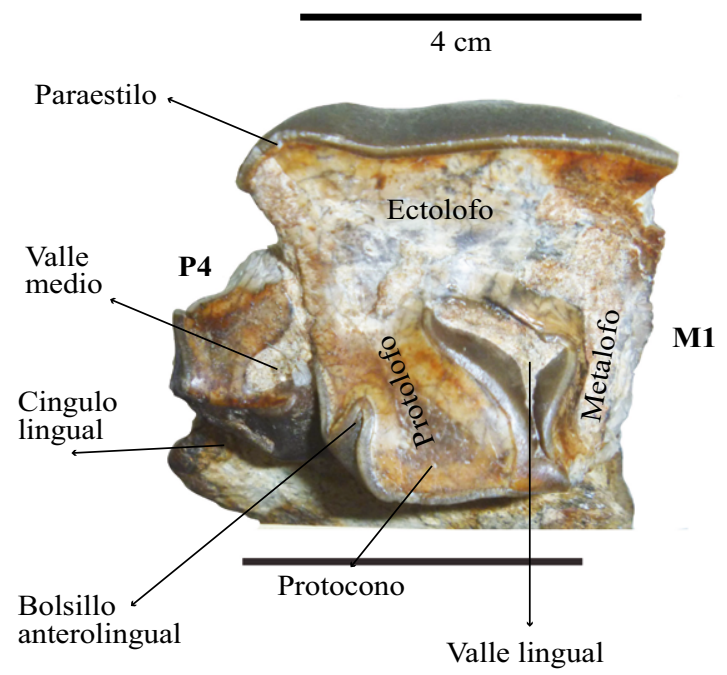

Figura 4. Vista oclusal del premolar (P4) y el molar (M1) superiores izquierdos
(Ameghino, 1901), Albertogaudrya (Ameghino, 1901; Carbajal, et al., 1977) y Maddenia (Kramarz \& Bond, 2009). En el lado lingual el cíngulo está presente, siendo mucho menos prominente que el que presenta $H$. castanedaii, y se reduce levemente hacia la mitad de su longitud encerrando dos leves depresiones ubicadas en las porciones anterior y posterior del diente, siendo la anterior menos profunda y más reducida que la posterior. En la especie $H$. castanedaii estas características son más pronunciadas y la parte anterior es propiamente un bolsillo antero-lingual, rasgo bastante reducido en el ejemplar descrito, y ausente en H. Miyou. El P4 tiene un amplio valle central con orientación antero-posterior y se ensancha hasta el borde anterior del M1, a diferencia del P4 de H. castanedaii, en el cual el valle central se abre diagonalmente hacia el lado lingual. Las raíces del P4 están incluidas en el maxilar, es decir no son visibles a pesar de la abrasión sufrida por el contacto con la arena de la playa y el agua de la ciénaga.

En la familia Atrapotheridae los molares superiores se caracterizan por la presencia de un valle central en forma de ' $\mathrm{Y}$ ', de abertura en el lado lingual. Sin embargo, el valle lingual del M1 del espécimen en estudio, si bien se dirige al lado lingual, está encerrado por un cíngulo lingual (borde superior del lado lingual del molar); posiblemente la abertura al lado lingual estuvo presente, pero debido al grado de desgaste desapareció. Los brazos internos de este valle en forma de ' $\mathrm{Y}$ ' están formados por un ganchillo muy corto, orientado hacia el metalofo, en tanto que el ganchillo más largo se orienta en dirección del protolofo. El M1 es birradicular, carece de pliegues en su lado lingual como en todos los Uruguaytheriinae, y el paraestilo reducido solo da lugar a una muy leve depresión en la parte anterior de la corona del lado labial. El M1 posee un profundo bolsillo anterolingual, característica presente en Xenastrapotherium, Granastrapotherium e Hilarcotherium. El bolsillo antero-lingual del M1 del espécimen estudiado es más agudo y tiene su base o silla menos pronunciada que la de $H$. castanedaii. El metalofo de este ejemplar está parcialmente relacionado con el borde lingual del protocono por el cíngulo que encierra el valle lingual, pero no conserva el cíngulo lingual en la base del metalofo, similar a lo observado en Hilarcotherium castanedaii, 
Granastrapotherium y Xenastrapotherium. El espécimen en estudio perdió el borde posterolingual y por ello no se aprecia si existió o no un metastilo.

Ya que el espécimen en estudio presenta algunas diferencias con Hilarcotherium castanedaii e Hilarcotherium miyou, se decidió designarlo tan solo a nivel genérico como Hilarcotherium sp., dada la falta de material complementario para comparar los restos. Asimismo, se consideró que la especie tipo está definida para este taxón a partir de un solo individuo, y que el material de H. miyou es fragmentario, por lo que se carece de paratipos o de material en diferentes estados de ontogenia que permitan erigir con certeza el material de estudio como una nueva especie o asignarla a alguna de las dos ya definidas.

\section{Material asociado}

Purussaurus neivensis. En la misma localidad, los señores Florián y Martínez encontraron un diente de caimán que por sus dimensiones y características parece corresponder a Purussaurus (Caimaninae). El diente tiene una corona con esmalte de 43,26 mm y gran parte de la raíz, lo que le da a la pieza un largo máximo de $92,56 \mathrm{~mm}$. El diente es ancho y romo y su sección basal es ligeramente ovalada (Figura 5A y B); en la superficie de la corona el esmalte es arrugado, siendo estas arrugas más pronunciadas a medida que se acercan a la parte apical del diente, pero menos marcadas hacia la base de la corona, en donde solo presenta finas estrías verticales.

El diente presenta dos carinas pronunciadas en los lados anterior y posterior, las cuales están presentes desde la parte media de la corona hasta la parte apical del diente: los bordes de estas estructuras tienen ornamentación fina como consecuencia de las arrugas de la corona, que se orientan tanto del lado lingual como del lado bucal en la parte media de la corona (Figura 5A y B) perpendicularmente al eje del diente, dando lugar a un borde arrugado. Esto concuerda con lo reportado por Moreno-Bernal, et al., 2016, para identificar y diferenciar a Purussaurus neivensis de Purussaurus mirandai.

Tortuga matamata (Chelus). El material de fósiles de tortugas matamata de la ciénaga de Zapatosa es bastante fragmentario. El recolectado en esta ocasión está en poder de los señores Florián y Martínez. En dicho material se distingue el género Chelus tan solo por los característicos mucrones o grandes prominencias antero-posteriores en sus caparazones. En futuras visitas tendría que hacerse un estudio detallado de este nuevo material y obtener mejores ejemplares que aporten datos a las discusiones sobre el parentesco existente entre las matamatas fósiles y las existentes en la actualidad, tema que los especialistas aún están estudiando.

Otras tortugas (familia Podocnemididae?). En el sitio del hallazgo se encontraron y recolectaron dos fragmentos de caparazones (o plastrones?) de quelonios. Dichos fragmentos son lisos, ligeramente convexos, con medidas que oscilan entre los 30 y los $20 \mathrm{~cm}$ de diámetro. Los restos se asignaron tentativamente a la familia Podocnemididae, pero como en el caso de las matamatas, es necesario que los especialistas estudien los restos de quelonios que se encuentran frecuentemente en algunas de las playas de la ciénaga de Zapatosa.

\section{Repositorio}

El astrapoterio estudiado corresponde al número de catálogo SGC-MGJRG.2018.V.2, en tanto que al diente de

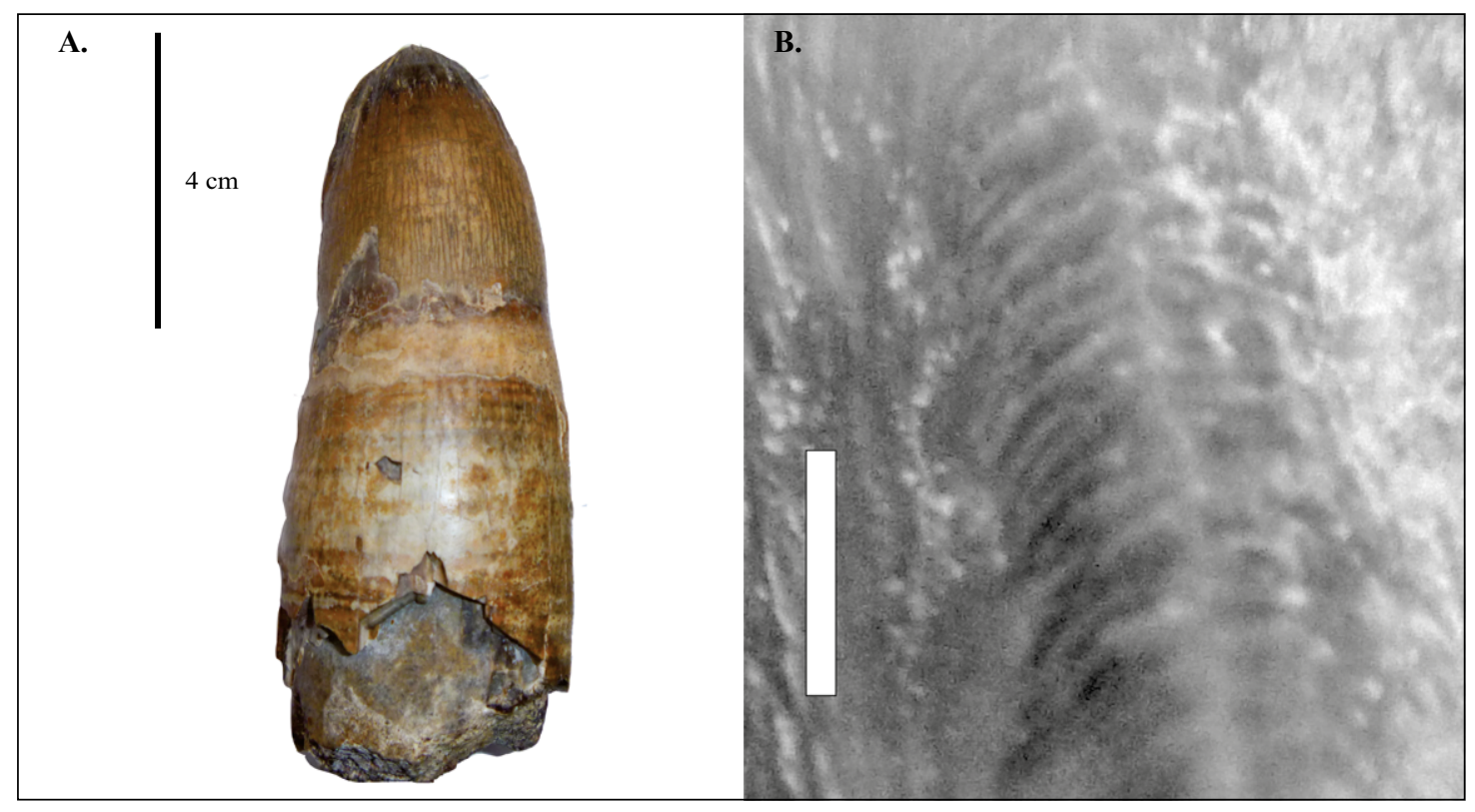

Figuras 5. A. Diente de Purussaurus neivensis (SGC-MGNJRG.2018.V.7) B. detalle en vista lateral de la carena que muestra la ornamentación arrugada propia de la especie (Moreno-Bernal, 2015); escala $2 \mathrm{~mm}$ 
Purussaurus le fue asignado el número de catálogo SGC. MGJRG.2018.V.7, y a los restos de tortugas Podocnemididae? los números SGC.MGJRG.2018.V.8 y SGC. MGJRG.2018.V.9. Las piezas reposan en la exhibición y las colecciones de fauna del Mioceno del Museo Geológico "José Royo y Gómez" del Servicio Geológico Colombiano en Bogotá.

\section{Discusión y conclusiones}

Aparte de los astrapoterios miocénicos del desierto de la Tatacoa (Villavieja, Huila) son pocas las descripciones de estos mamíferos placentarios en otros lugares del territorio colombiano. Entre los hallados se destacan Hilarcotherium castanedaii de la Formación La Victoria (Grupo Honda) en el municipio de Purificación (Tolima) y Hilarcotherium miyou de la Formación Castilletes, en la cuenca de Cocinetas, al sureste de la península de La Guajira. Así el hallazgo de los restos de especímenes de este género en la ciénaga de Zapatosa supone la ampliación paleobiogeográfica de la distribución de Hilarcotherium, y aporta datos que permiten mejorar los procesos e interpretaciones cladísticas entre los Uruguaytheriinae.

Este nuevo registro de material de mamíferos y reptiles constituye un recurso importante para conocer la distribución y las relaciones existentes entre las faunas del Mioceno, además de constituir un elemento importante para mejorar y refinar la cartografía geológica de la región. Para tal fin se hace necesario adelantar campañas de campo en tiempo seco que permitan recuperar y estudiar nuevo material. Así se podrán conocer mejor las relaciones entre las faunas de los yacimientos de las regiones del valle superior y el valle inferior del Magdalena, el sureste de La Guajira e, incluso, su continuación estratigráfica hacia Venezuela.

Se hace necesario que los investigadores especialistas en reptiles (Crocodylia y Testudines) y otros expertos en paloentología se involucren en el estudio del material que eventualmente se recupere en esta región del país. Esta clase de hallazgos constituyen, además, una excelente oportunidad para fortalecer las relaciones interinstitucionales, pues brinda un espacio para adelantar proyectos conjuntos con diferentes entidades que trabajan en el ámbito de las geociencias.

\section{Agradecimientos}

A los señores Hasmet Florián y Luis Martínez por el acompañamiento hasta el lugar del hallazgo y la generosa donación de las piezas que recolectaron. A Jorge Moreno Bernal por sus valiosos comentarios para la descripción del diente de caimán. A Leopoldo González Oviedo, Coordinador del Grupo de Trabajo de Museo e Investigaciones Asociadas del Servicio Geológico Colombiano.

\section{Referencias}

Ameghino, F. (1887). Enumeración sistemática de las especies de mamíferos fósiles coleccionados por Carlos Ameghino en los terrenos eocenos de Patagonia Austral y depositados en el Museo de La Plata. Boletín del Museo de La Plata. 1: 1-26.
Ameghino, F. (1901). Notices préliminaires sur des ongulés nouveaux des terrains crétacés de Patagonie [Preliminary notes on new ungulates from the Cretaceous terrains of Patagonia]. Boletín de la Academia Nacional de Ciencias de Córdoba. 16: 349-429.

Ameghino, F. (1904). Nuevas especies de mamíferos, cretáceos y terciarios de la República Argentina [New species of mammals, Cretaceous and Tertiarty, from the Argentine Republic]. Anales de la Sociedad Científica Argentina. 5658: 1-142.

Carbajal, E., Pascual, R., Pinedo, R., Salfity, J.A., Vucetich, M. G. (1977). Un nuevo mamífero de la Formación Lumbrera (Grupo Salta) de la comarca de Carahuasi (Salta, Argentina). Edad y correlaciones. Publicaciones del Museo Municipal de Ciencias Naturales de Mar del Plata "Lorenzo Scaglia”. 2 (7):148-163

Carrillo-Sánchez, J. D., Amson, E., Jaramillo, C., Sanchéz, R., Quiroz, L., Cuartas, C., Rincón, A.F., Sánchez-Villagra, M. F. (2018). The Neogene Record of Nothern South American Native Ungulates. Smithsonian contributions to paleobiology, No. 101. 0081-0266. Washington, D.C.: Smithsonian Institution Scholarly Press, 2018.

Head, J., Bloch, J., Hastings, A., Bourke, J., Cadena, E., Herrera, F., Polly, D., Jaramillo, C. (2009). Giant boid snake from the Paleocene Neotropics reveals hotter past equatorial temperatures. Nature. 457: 715-718. Doi: 10. 1038/nature07671

Ingeominas-UIS. (2006). Geología de la Plancha 55 El Banco, escala 1:100.000, (Acuerdo específico 004 de 2005 complementario al convenio marco de cooperación 005 celebrado entre INGEOMINAS y la Universidad Industrial de Santander (UIS)). Bogotá, Colombia.

Jaramillo, C., Moreno, F., Hendy, A. J., Sánchez-Villagra, M. R., Marty, D. (2015). Preface: La Guajira, Colombia: A new window into the Cenozoic neotropical biodiversity and the Great American Biotic Interchange. Swiss Journal of Paleontology. 134 (1):1-4.

Johnson, S. C. \& Madden, R. H. (1997). Uruguaytheriinae Astrapotheres of Tropical South America. Chapter 22 in "Vertebrate Paleontology in the Neotropics. The Miocene Fauna of La Venta, Colombia". Edited by Richard F. Kay, Richard H. Madden, Richard L. Cifelli, and John J. Flynn. Smithsonian Institution Press. Washington and London.

Johnson, S. C. (1984). Astrapotheres from the Miocene of Colombia, South America. University of California. Dissertation submitted in partial satisfaction of the requirements for the degree of Doctor of Philosophy in Paleontology in the Graduate Division of the University of California, Berkeley.

Kraglievich, L. (1928). Sobre el supuesto Astrapotherium christi Stehlin, descubierto en Venezuela (Xenastrapotherium n. gen.) y sus relaciones con Astrapotherium magnum y Uruguaytherium beaulieui.. Editorial Franco-Argentina. Buenos Aires. p.- 1-16.

Kramarz, A. G. \& Bond, M. (2009). A new Oligocene astrapothere (Mammalia, Meridungulata) from Patagonia and a new appraisal of astrapothere phylogeny. Journal of Systematic Palaeontology. 7: 117-128.

Kramarz, A. G., \& Bond, M. (2011). A new early Miocene astrapotheriid (Mammalia, Astrapotheria) from Northern Patagonia, Argentina. NeuesJahrbuch fur Geologie und Palaontologie-Abhandlungen. 260: 277-287. 
Lydekker, R. (1894). Contribution to the knowledge of the fossil vertebrates of Argentina. 3. Study of extinct Argentine ungulates. Anales del Museo de La Plata, Paleontología Argentina. 2: 1-91, pls 1-32.

Moreno-Bernal, J. W., Head, J., Jaramillo, C.A. (2016). Fossil crocodilians from High Guajira Peninsula of Colombia: Neogene faunal change in nothernmost South America. Journal of Vertebrate Paleontology. Doi:10.1080/0272463 4.2016.1110586

Moreno, F., Hendy, A., Quiroz, l., Hoyos, N., Jones, D., Zapata, V., Zapata, S., Ballen, G., Cadena, E., Cárdenas, A., Carrillo, J., Delgado-Sierra, D., Escobar, J., Martínez, I., Martínez, C., Montes, C., Moreno, J., Pérez, N., Sánchez, R., Suárez, C., Vallejo-Pareja, C., Jaramillo, C. (2015). Revised stratigraphy of Neogene strata in the Cocinetas Basin, La Guajira, Colombia. Swiss Journal of Palaeontoly. 134: 5-43.
Suárez, C., Forasiepi, A. M., Goin, F.J., Jaramillo, C. (2016). Insights into the Neotropics prior to the Great American Biotic Interchange: New Evidence of Mammalian Predators from the Miocene of Northern Colombia. Journal of Vertebrate Paleontology. 36 (1): e1029581. Doi:10.1080/02 724634.2015.1029581

Vallejo-Pareja, M. C., Carrillo, J.D., Moreno-Bernal, J.W., Pardo-Jaramillo, M., Rodríguez-González, D.F., MuñozDurán, J. (2015). Hilarcotherium castanedaii, gen. et sp. nov., A New Miocene Astrapothere (Mammalia, Astrapotheriidae) From The Upper Magdalena Valley, Colombia. Journal of Vertebrate Paleontology. 35: e903960. Doi: $10.1080 / 02724634.2014 .903960$.

Villarroel, C. \& Clavijo, J. (2005). Los mamiferos fósiles y las edades de las sedimentitas continentales del Neógeno de la costa Caribe colombiana. Revista de la Academia Colombiana de Ciencias Exactas, Fisicas y Naturales. 29 (112): 345-356. 